\title{
A New Approach for Estimating High-Speed Analog-to-Digital Converter Error
}

\author{
Galia D. Muginov and Anastasios N. Venetsanopoulos, Fellow, IEEE
}

\begin{abstract}
One of the most significant types of error in digital signal processing (DSP) systems working with wideband signals is the error introduced by the analog-to-digital converter (ADC). This paper investigates an accurate, simple, and low-cost method, which can be used for calibrating, testing, and quick-monitoring ADC. The proposed method analyzes the deviation of a value of the converted signal, which is similar to the ADC working signal, from its true value. The deviation represents the tested ADC error evaluated for both frequency and voltage ranges of ADC operations using statistical data processing. It gives the only necessary ADC accuracy characteristic to a user leaving the rest to the designer. Comparisons between the proposed ADC testing approach and the known techniques are provided. The errors incurred by the method are analyzed. A special source of random signal with controlled statistical parameters and its calibration technique are also described.
\end{abstract}

Index Terms-Analog-to-digital converter (ADC), digital signal processing (DSP), error, evaluation, high-speed ADC, random signal, test, working conditions.

\section{INTRODUCTION}

$\mathbf{K}$ NOWN analog-to-digital converter (ADC) testing techniques deal with the evaluation of many accuracy characteristics (AC) of the converter but the conversion error of any value of an ADC input signal in working conditions, i.e., the ADC error, is not evaluated. This observation is supported by an IEEE standard [1], which is providing the globally accepted methods for measuring numerous ADC specifications without specifying the ADC error [1]. However, there are publications dedicated to automatic ADC testing and aimed at the ADC error evaluation [2]-[7]. In these references, it is recognized that the most difficult aspect of testing is the test signal source organization. In [3] "stepped input changes" are used in dynamic ADC testing. In [4], a sinewave is accepted for dynamic operating conditions. In [5], a maximum length sequence (M-sequence) is suggested in static testing and in [6] it is used in dynamic testing for special data acquisition and data processing based on Walsh functions. In [7], a complex signal with controlled statistical parameters is formed from the M-sequence. This signal is similar to ADC working signals.

In modern production of IC's (ADC is one of them), the industry has found that the costs of test equipment, time to test and test procedures are the dominating manufacturing

Manuscript received June 3, 1996.

G. D. Muginov is with the Department of Electrical and Computer Engineering, University of Toronto, Toronto, Ont., Canada, M5S 1A4.

A. N. Venetsanopoulos is with the Department of Electrical and Computer Engineering, University of Toronto, Toronto, Ont., Canada M5S 3G4 (e-mail: anv@dsp.toronto.edu).

Publisher Item Identifier S 0018-9456(97)06502-9. costs. There are publications which suggest methods in order to simplify the procedures and to shorten the time for finding some AC in dynamic testing of IC's [8].

The proposed testing approach takes into account the user and industry needs and provides automatic evaluation of the ADC error in static and dynamic actual operating conditions. It follows the global strategy described in [2], [3], [6], [7], applies the test signal from [7], but introduces a new data acquisition mode and statistical data processing.

\section{General APPROACH AND PRINCIPLE}

In an $\mathrm{ADC}$ manual [9], the $\mathrm{AC}$ are represented by their transfer characteristics and dynamic characteristics.

If the highest frequency $f_{H}$ of the converted signal is significantly lower than the frequency $f_{\text {conv }}$ which can be converted by ADC, the ADC input signal may be considered as a constant. $\mathrm{AC}$ for $f_{H} \ll f_{\mathrm{Conv}}$ are transfer characteristics which characterize the systematic component of the ADC error for these operations.

For a higher frequency of the converted signal, or for $f_{H} \leq$ $f_{\text {Conv }}, \mathrm{AC}$ are dynamic characteristics which characterize the random and systematic components of the ADC error for these operations.

All AC specified in the manual contribute to the ADC error. Therefore the ADC error may be calculated using these AC. Obtaining the ADC error in such a way may be considered as an indirect way because this error is calculated after direct evaluation of the AC. Indirect calculation of the ADC error contains significant approximation errors. That is why the ADC error obtained is not useful for an evaluation of the DSP error.

The new testing approach uses the direct way for determining the ADC error which may be described as

$$
\Delta_{\mathrm{ADC}}=y_{\text {real, for } f_{H} \ll f_{\text {conv }}}-x_{\text {true }} \text { for } f_{H} \ll f_{\text {conv }}
$$

and

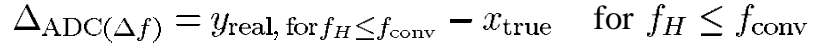

where $x_{\text {true }}$ is a test signal quantity which is received by the most accurate measurement, $y_{\text {real, for } f_{H} \ll f_{\text {conv }}}$ and $y_{\text {real }}$ for $f_{H} \leq f_{\text {conv }}$ are the ADC corresponding responses, measured in static and dynamic operations. The quality of such an ADC error evaluation depends on accuracy of $y_{\text {real }}$ and $x_{\text {true }}$ measurement only which can be sufficiently high.

The main idea of the method is as follows. A scale is used for the representation of variables of a signal. When the signal runs slowly enough, an accurate voltmeter can be used and the 


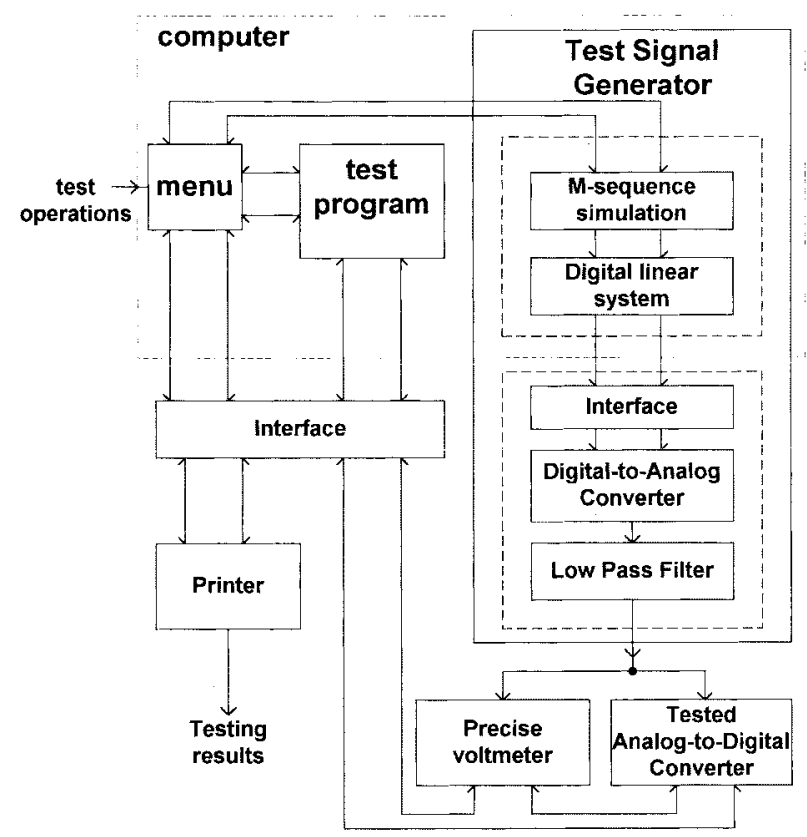

Fig. 1. Distortion test circuit for testing high speed ADC's in static and dynamic operations by the proposed method.

variables, converted by the tested ADC simultaneously with the voltmeter readings, are compared. From this comparison the real ADC response function is obtained, the ADC error for $f_{H} \ll f_{\text {conv }}$ is determined and then the transfer characteristics are calculated. When the signal frequency increases, the time axis is compressed and the signal varies faster over each variable. The same variables are converted by ADC again and compared with their estimates for $f_{H} \ll f_{\text {conv }}$. The ADC error for $f_{H} \leq f_{\text {conv }}$ is determined from this comparison.

\section{Choice of the Test Signal}

The following requirements are considered for choosing the variables scale of the test signal, or test variables.

1) The maximum spacing between neighboring quantities $\Delta x_{\text {interp. }}$ must be chosen taking into account the ADC response function interpolation error. This spacing defines the minimum number $N_{\text {interp. }}$ of variables within the voltage range on the function.

2) The minimum test variables separation time must be longer or equal to the $\mathrm{ADC}$ conversion time for the highest frequency $f_{H}$ in order to avoid dropping out some test variables from the scale.

3) The test signal reproduction error should be minimum.

The test signal initial model is an M-sequence with a period $L$ of nonrepeating binary numbers. An analog signal realization is obtained after passing this sequence through a digital linear system, a digital-to-analog converter (DAC) and a lowpass filter (LPF) (see test signal generator (TSG) in Fig. 1). Any test variable can be calculated and reproduced in a fixed order of specific times $k_{1} t_{\text {clock }}$ where $k_{1}=0,1,2 \ldots(L-$ $1) ; t_{\text {clock }}$ is the time interval between neighboring numbers. The amplitude-frequency-time parameters of such a signal are known and controlled. The signal period is $T=t_{\text {clock }} L$. By changing $t_{\text {clock }}$, the signal realization can be "compressed" or

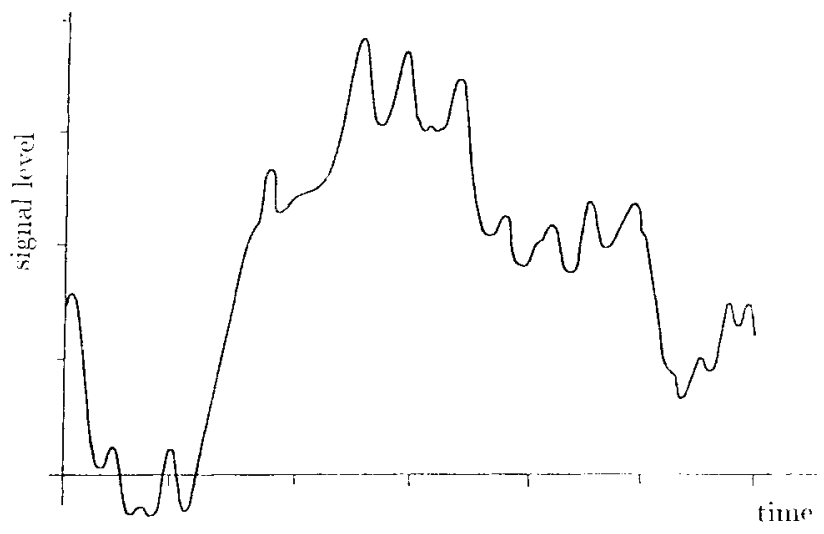

(a)

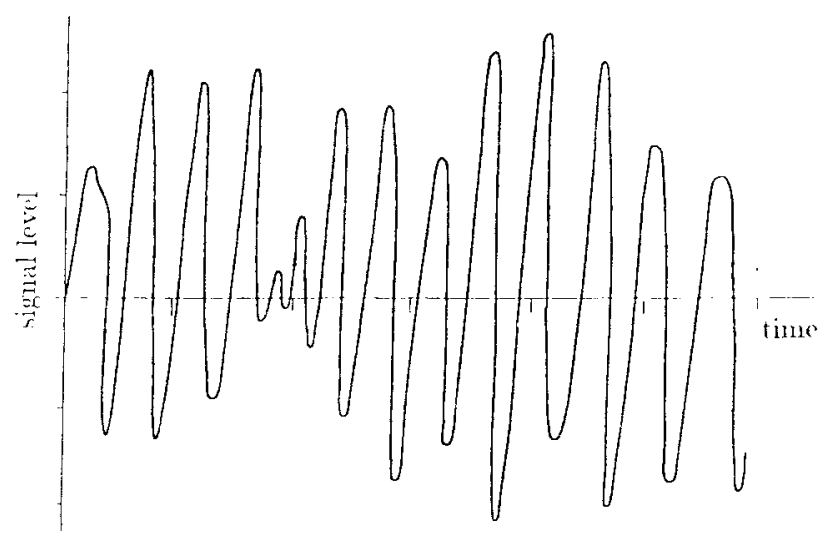

(b)

Fig. 2. Realizations of signals with Gaussian probability density and correlation functions $R_{x x}(\tau)=\sigma^{2} \exp ^{-\alpha|\tau|}$ (a) and $R_{x x}(\tau)=\sigma^{2} \exp -\alpha|\tau| \cos \omega_{o} \tau$ for $\frac{\omega_{0}}{\alpha} \simeq 7.9$ (b) at the output of test signal generator in Fig. 1.

"stretched," i.e., its bandwidth is changed without changing the signal waveform. The minimum difference between the signal quantities is $\Delta x=\frac{x_{\text {nom }}}{L}$. The conditions $\Delta x_{\text {interp. }} \gg \Delta x$, and $N_{\text {interp. }} \ll L$ suffice for practically any set interpolation error as $L$ is of the order $10^{6}$. The test variables scale of this signal satisfies the above requirements. Once the test variables are determined, their ordinal numbers in the M-sequence must be stored in memory.

The advantages of the algorithm are the similarity of the simulated signal to the ADC working signals in all frequency ranges and the flexibility of signal parameters. Two realizations of such a signal, simulated by TSG shown in Fig. 1, are shown in Fig. 2.

\section{PRocedures}

For data processing the matrix is used

$$
[A]=\left[\begin{array}{ccccc}
\hat{A}_{11} & \cdots & \hat{A}_{1 j} & \cdots & \hat{A}_{1 N} \\
\vdots & \vdots & \vdots & & \\
\hat{A}_{i 1} & \cdots & \hat{A}_{i j} & \cdots & \hat{A}_{i N} \\
\vdots & & \vdots & \vdots & \\
\hat{A}_{M 1} & \cdots & \hat{A}_{M j} & \cdots & \hat{A}_{M N}
\end{array}\right]
$$


where $i=1,2 \ldots M ; M$ is the sample size for the ADC error estimation; $j=1, \ldots q, \ldots p, \ldots N ; N$ is the number of the test variables $\left(N \geq N_{\text {interp. }}\right.$, for ADC calibration $N=2^{R}$ where $R$ is the ADC resolution); $\hat{A}_{i j}$ is the $i$ th reading of the $j$ th test variable.

For $f_{H} \ll f_{\text {conv }}$ each $\hat{A}_{i j}$ obtained at the tested ADC output, $\hat{A}_{i j \mathrm{ADC}}$, is made up as follows:

$$
\hat{A}_{i j \mathrm{ADC}}=A_{j \mathrm{TSG}}+\Delta_{i j \mathrm{ADC}}+\Delta_{i j \mathrm{TSG}}
$$

where $A_{j \mathrm{TSG}}$ is the ideal $j$ th test variable; $\Delta_{i j \mathrm{ADC}}$ and $\Delta_{i j \mathrm{TSG}}$ are the ADC error and the TSG error for the $j$ th test variable in its $i$ th reading. Simultaneously, at the voltmeter output

$$
\hat{A}_{i j V}=A_{j \mathrm{TSG}}+\Delta_{i j \mathrm{TSG}}+\Delta_{i j V}
$$

where $\Delta_{i j V}$ is the voltmeter error for the $j$ th test variable in the $i$ th reading. An accurate enough voltmeter must be used, so that its error may be neglected in solving this problem. Therefore

$$
\Delta_{i j V}=0 .
$$

For $M \geq 50$ it is possible to neglect the statistical scattering of the estimates of the sample means of $\hat{A}_{i j \mathrm{ADC}}$ and $\hat{A}_{i j \mathrm{~V}}$ and to accept

$$
\begin{aligned}
m_{j \mathrm{ADC}} & =\frac{1}{M} \sum_{i=1}^{M} \hat{A}_{i j \mathrm{ADC}} \\
& =A_{j \mathrm{TSG}}+\Delta_{\mathrm{syst} . j \mathrm{ADC}}+\Delta_{\text {syst. } j \mathrm{TSG}} \\
m_{j V} & =\frac{1}{M} \sum_{i=1}^{M} \hat{A}_{i j V} \\
& =A_{j \mathrm{TSG}}+\Delta_{\text {syst. } j \mathrm{TSG}}=m_{j \mathrm{TSG}}
\end{aligned}
$$

where $\Delta_{\text {syst. } j \text { ADC }}$ and $\Delta_{\text {syst. } j \text { TSG }}$ are the systematic components of the ADC and TSG errors. The value $m_{j V}$, or $m_{j \mathrm{TSG}}$, is considered as the true $j$ th quantity of the ADC response function and $m_{j \mathrm{ADC}}$ as its real $j$ th quantity. The maximum random component of the ADC error obtained for $j$ th quantity of the ADC response function is

$$
\begin{aligned}
\Delta_{\text {rand } . j \mathrm{ADC}} & = \pm\left|\hat{A}_{i j \mathrm{ADC}}-\hat{A}_{i j V}-m_{j \mathrm{ADC}}\right|_{\max } \\
& = \pm\left|\Delta_{\text {rand. } . i j \mathrm{ADC}}\right|_{\max }
\end{aligned}
$$

and the systematic component for the same quantity is

$$
\Delta_{\mathrm{syst} . j \mathrm{ADC}}=m_{j \mathrm{ADC}}-m_{j V} .
$$

As is seen from (7) and (8), for $f_{H} \ll f_{\text {conv }}$ the TSG error does not influence the ADC error.

Now the ADC response function for $f_{H} \ll f_{\text {conv }}$ can be plotted in the following way. The sample means $m_{j V}$ are placed on the horizontal axis and the sample means $m_{j \mathrm{ADC}}$ are placed on the vertical axis. So curve 2 is obtained in Fig. 3. Calculation of the AC specified in the ADC certificate is presented in the Appendix.

The next step is finding the ADC error for $f_{H} \leq f_{\text {conv }}$. For the tested frequency range $\Delta f_{k}$, where $k=1 \cdots N_{f}$, the matrix (1) consists of $\hat{A}_{i j \mathrm{ADC}\left(\Delta f_{k}\right)}$, which are the same test

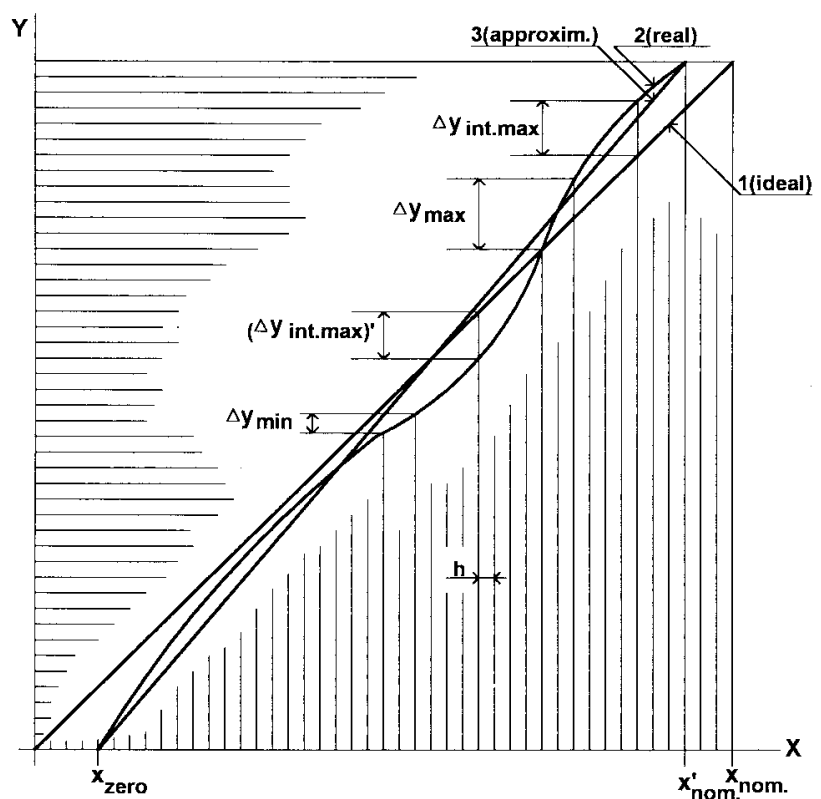

Fig. 3. ADC response function. Clarification of transfer characteristics.

variables as above, but now they are in the faster running test signal. By analogy with (2), (5), (7), and (8) it may be written

$$
\begin{aligned}
& \hat{A}_{i j \mathrm{ADC}\left(\Delta f_{k}\right)}=A_{j \mathrm{TSG}}+\Delta_{i j \mathrm{ADC}\left(\Delta f_{k}\right)}+\Delta_{i j \mathrm{TSG}\left(\Delta f_{k}\right)} \\
& m_{j \mathrm{ADC}\left(\Delta f_{k}\right)}=A_{j \mathrm{TSG}}+\Delta_{\text {syst. } j \mathrm{ADC}\left(\Delta f_{k}\right)} \\
& +\Delta_{\text {syst. } . j \operatorname{TSG}\left(\Delta f_{k}\right)} \\
& \Delta_{\text {rand } j \mathrm{ADC}\left(\Delta f_{k}\right)}= \pm\left|\hat{A}_{i j \mathrm{ADC}\left(\Delta f_{k}\right)}-m_{j \mathrm{ADC}\left(\Delta f_{k}\right)}\right|_{\max } \\
& = \pm \mid \Delta_{\text {rand }, i j \operatorname{ADC}\left(\Delta f_{k}\right)} \\
& +\left.\Delta_{\text {rand. } i j \mathrm{TSG}\left(\Delta f_{k}\right)}\right|_{\max } \\
& \Delta_{\text {syst. } j \mathrm{ADC}\left(\Delta f_{k}\right)}^{\prime}=m_{j \mathrm{ADC}\left(\Delta f_{k}\right)}-m_{j V} \\
& =\Delta_{\text {syst. } j \mathrm{ADC}\left(\Delta f_{k}\right)}+\left(\Delta_{\Delta_{\mathrm{syst} . j \mathrm{TSG}}}\right)_{\Delta f_{k}}
\end{aligned}
$$

where $\left(\Delta_{\Delta_{\text {syst }, j \mathrm{TSG}}}\right)_{\Delta_{f}}$ is variation of $\Delta_{\text {syst. } j \mathrm{TSG}}$ with frequency and $\Delta_{\text {rand. } i j \operatorname{TSG}\left(\Delta f_{k}\right)}$ is the TSG error random component for $j$ th test variable in $i$ th reading.

As is seen from (9) and (10), for $f_{H} \leq f_{\text {conv }}$ the systematic component of the TSG error does not influence the ADC error as well as for $f_{H} \ll f_{\text {conv }}$, but the changes of this component with frequency and the random component of the TSG error are involved in the ADC error.

The ADC error obtained is a function of frequency and voltage ranges and is represented by nomograms in the most common case.

\section{Method ERrors}

\section{A. General Approach}

The only distortion test circuit (DTC) shown in Fig. 1 contributes in the new approach error $\Delta_{\text {test new }}$ and allows to determine the ADC error directly. The new approach DTC is similar to the known technique DTC used in dynamic testing but synthesizers and LPF's are replaced by TSG. 
TABLE I

Main Sources of the Test Signal Generator ERror

\begin{tabular}{|c|c|c|}
\hline TSG entor sources & $\begin{array}{l}\text { Firor } \\
\text { dharacter }\end{array}$ & $\begin{array}{l}\text { Dependence } \\
\text { on freguenes }\end{array}$ \\
\hline \multicolumn{3}{|l|}{ 1. $D A C$ of $T S G$} \\
\hline a. Reference volt age variations & ranklom & independent \\
\hline b. R-2R ladder innperfection & systematic & inclependent \\
\hline c. Switchs imperfection & systematic & dependent \\
\hline \multicolumn{3}{|l|}{ 2.LPF of TSG } \\
\hline a. Attemation in the stopland & systemalic & dependent \\
\hline 1). Ripple in the passband & systematic & dependent \\
\hline \multicolumn{3}{|l|}{ 3.Simulation algorithm } \\
\hline Limited memory of digital & & \\
\hline linear system & sustematic & independe \\
\hline \multicolumn{3}{|l|}{ 4.Data processing algorithm } \\
\hline Finite sample size & systematic & inclepender \\
\hline
\end{tabular}

The known ADC testing techniques are realized by some DTC's, whose number is usually not more than three. As the ADC error may be calculated indirectly in this case, each of the DTC's contributes in the ADC tested error through the AC estimated before. The indirect calculation of the ADC error, implemented with some assumptions and restrictions, also contribute to the known technique error $\Delta_{\text {test known. Taking }}$ into account these facts, it is possible to accept

$$
\Delta_{\text {test new }}<\Delta_{\text {test known }} .
$$

\section{B. Analysis}

For $f_{H} \ll f_{\text {conv }}$, the new approach accuracy is only determined by the voltmeter accuracy as the method procedures eliminate $\Delta_{\text {TSG }}$ from the ADC error [see (7) and (8)]. The modern voltmeter error may be $0.001 \%$. Today it is enough to observe condition (4) for evaluating even the advanced ADC error. The accuracy of the method may be estimated by

$$
\Delta_{\text {test new } f_{H} \ll f_{\text {conv }}}=\Delta_{V} .
$$

For $f_{H} \leq f_{\text {conv }}, \Delta_{\text {test.new }}$ depends on the random component of the TSG error and on the variations of its systematic component [see (9) and (10)] and may be written as

$\Delta_{\text {test new } f_{H} \leq f_{\text {conv }}}=F_{\text {new }}\left[\left(\Delta_{\text {rand.TSG }}\right)_{\Delta f_{k}},\left(\Delta_{\Delta_{\text {syst TSG }}}\right)_{\Delta f_{k}}\right]$.

Considering sources of the TSG error, shown in Table I, it is possible to accept

$$
\left(\Delta_{\text {rand.TSG }}\right)_{\Delta f_{k}}=\Delta_{\text {rand.TSG }}
$$

where $\Delta_{\text {rand.TSG }}$ is a characteristic of the statistics found using (3) and (6) as

$$
\Delta_{\text {rand. } i j \mathrm{TSG}}=\hat{A}_{i j V}-m_{j V} .
$$

Three sources from Table I, contributing in $\Delta_{\text {test new } f_{H} \leq f_{\text {conv }}}$ through $\left(\Delta_{\Delta_{\text {svst.TSG }}}\right)_{\Delta f_{k}}$, must be discussed in $1 \mathrm{c}, 2 \mathrm{a}, 2 \mathrm{~b}$ (see Table I).

The factor 1c may give a significant contribution depending on the relationship between the switch response and the frequency range of the signal at its input. In the new approach this factor influence is minimized by using the same test variables for different $\Delta f_{k}$ and observing the condition

$$
T_{\text {settl.DAC for all procedures }}=T_{\text {settl.DACmin }}=\text { const }
$$

where $T_{\text {settl.DAC }}$ is the settling time of the DAC used in TSG for all frequency ranges. Considering (13) and that the maximum DAC frequency is about two orders higher than that in the ADC, the factor 1c contribution may be neglected.

Contributions of the factors $2 \mathrm{a}$ and $2 \mathrm{~b}$ are the most significant. The LPF of TSG, used for eliminating the frequency $f_{\text {clock }}=\frac{1}{t_{\text {clock }}}$ from the test signal, changes the test signal spectrum. The smaller the part of the test signal spectrum located nearby $f_{\text {clock }}$ is, the smaller this change is. In the approach for all frequency ranges, the condition observed is

$$
f_{\text {clock }}=k_{2} f_{H}
$$

with $k_{2}=10$. The stopband start frequency is equal $f_{\text {clock }}$. The TSG digital linear system taking into account (14) locates $95 \%$ of the test signal spectrum inside $0.1 \%$ of the passband and minimize the influence of the factor $2 \mathrm{a}$.

The factor $2 \mathrm{~b}$ cannot be minimized by the method. This factor is specified for the commercial LPF [10] and evaluated with sufficient accuracy by LPF testing techniques. There are LPF's having possibilities for outside connections for getting different frequency ranges. In this case, using the known LPF testing techniques, the ripple is evaluated for each $f_{k}$. Then $\left(\Delta_{\Delta_{\text {syst.TSG }}}\right)_{\Delta f_{k}}$ may be found as

$$
\left(\Delta_{\Delta_{\text {syst,TSG }}}\right)_{\Delta f_{k}}=\left(m_{j \mathrm{TSG}}\right)_{\max }\left(K_{\text {ripp } f_{k}}-K_{\text {ripp. }}\right)
$$

where $K_{\text {ripp. } f_{k}}$ and $K_{\text {ripp. }}$ are the maximum LPF response gains in the frequency range $f_{k}$ and in the frequency range used in static testing. The value, found from (15), turns out to be excessive. Finally, considering (11) and (15)

$$
\begin{aligned}
& \left(\Delta_{\text {test new }}\right)_{f_{k}} \\
& \quad \leq\left|\left(\Delta_{\text {rand.TSG }}\right)\right|+\left|m_{j \text { TSG }}\right|_{\text {max }}\left(K_{\text {ripp } . f_{k}}-K_{\text {ripp. }}\right) .
\end{aligned}
$$

Decreasing $\Delta_{\text {test new } f_{H} \leq f_{\text {conv }}}$ may be reached by the careful tuning up of a commercial LPF or by a special LPF design, e.g., the second-order Butterworth LPF.

\section{The TSG Calibration}

The TSG must be calibrated for testing each ADC type, at least. The reason is that the test variable set depends on the $\mathrm{ADC}$ frequency range, on the plotting accuracy of the ADC tested response function and on the ADC working signal area. If the synthesizer of the known techniques is used as TSG, it must be calibrated in the same way as TSG in order to obtain the test variable set and the synthesizer error, which is not included in its certificate. The TSG calibration has three steps.

The first step is the evaluation of ripple $K_{\text {ripp. }}$ and $K_{\text {ripp. } f_{k}}$ for each frequency range $f_{k}$ of the ADC tested by the known LPF testing techniques, if the LPS commercial specifications are not enough.

The second step is the evaluation of $\Delta_{\text {rand.TSG }}$ by the way described in Section IV and using appropriate data processing for statistics from (12). In this step, the range of $\left(m_{j \mathrm{TSG}}\right)$ [see (6)] is found. 


$$
\begin{aligned}
n_{\text {zeroLSB }}= & \frac{m_{q V}}{m_{N V}} 2^{R} \quad n_{\text {gainLSB }}=\frac{m_{N V}-m_{p V}}{m_{N V}} 2^{R} \\
n_{\text {int.LSB }}= & \mid\left(m_{j \mathrm{ADC}}-\left(m_{j V}-m_{q V}\right) \frac{m_{p \mathrm{ADC}}}{m_{p V}-m_{q V}}\right. \\
& \left.+\Delta_{\text {rand. } i j \mathrm{ADC}}\right)\left._{\max }\right|_{\max } \times \frac{2^{R}}{m_{N V}} \\
n_{\text {diff.LSB }}= & \frac{\left|A_{j \mathrm{ADC}}-A_{(j-1) \mathrm{ADC}}\right|_{\max }-\left|A_{j \mathrm{ADC}}-A_{(j-1) \mathrm{ADC}}\right|_{\min }}{m_{N V}} 2^{R}
\end{aligned}
$$

The third step is the calculation of the set of $\Delta_{\text {test new } f_{k}}$ using (16) and making decision about possibility to use the TSG signal as a model.

\section{CONCLUSION}

An innovative method for testing high speed ADC has been described. Its main characteristics are as follows.

1) The ADC error, found by the new approach, is the nearest to the real ADC error as it is obtained for the actual ADC environment.

2) The ADC error obtained allows the user to calculate his DSP error expected and choose optimum ADC operating conditions.

3) The error incurred by the new approach is smaller than the error incurred by the known techniques for testing high speed ADC's.

4) The new test cost is less than the known one as the procedures are automated, the time required is less and the equipment used is simpler.

The method features have been achieved by applying a classical method for estimating an error, known as "comparing to a measure," followed by statistical data processing. The measure is the particular sequence of the test signal variables estimated by a precise voltmeter. Practical realization of the method needs a PC with a built-in relay board, a precise programmable voltmeter, and a special program package.

The new approach may be used also for testing another circuit or electronic equipment working with complex signals. In such cases the concept developed is applied but with modified data processing.

\section{APPENDIX \\ CALCulation of ADC TRANSFER Characteristics}

The transfer characteristics, which characterize the systematic component of the ADC error, are provided in LSB units [9], composed by the differential linearity $\left(n_{\text {diff.LSB }}\right)$, the integral linearity ( $\left.n_{\text {int.LSB }}\right)$ the gain error $\left(n_{\text {gainLSB }}\right)$ and the zero error $\left(n_{\text {zeroLSB }}\right)$ and written as

$$
\begin{aligned}
& n_{\text {zeroLSB }}=\frac{x_{\text {zero }}}{h}, \quad n_{\text {gainLSB }}=\frac{x_{\text {nom }}-x_{\text {nom }}^{\prime}}{h} \\
& n_{\text {int.LSB }}=\frac{\left|\Delta y_{\text {int. max }}\right|}{h}, \quad n_{\text {diff.LSB }}=\frac{\left|\Delta y_{\text {max }}\right|-\left|\Delta y_{\text {min }}\right|}{h}
\end{aligned}
$$

where $\Delta y_{\max }, \Delta y_{\min }, \Delta y_{\text {int. } \max }, x_{\text {nom }}, x_{\text {nom }}^{\prime}, x_{\text {zero }}$ and $h=\frac{x_{\text {nom }}}{2^{R}}$ are shown in Fig. 3 .

If $m_{j \mathrm{ADC}}=\mathrm{const}$ for $j=N, N-1, \ldots p$ and if $m_{j \mathrm{ADC}}=$ var for $j=p-1, \ldots q+1$, then

$$
x_{\text {nom }}=m_{N V} \quad x_{\text {nom }}^{\prime}=m_{p V}
$$

If $m_{j \mathrm{ADC}}=0$ for $j=1.2, \ldots q$ and if $m_{j \mathrm{ADC}} \neq 0$ for $j=q+1, \ldots N$, then

$$
x_{\mathrm{zero}}=m_{q V} .
$$

The $\Delta y_{\text {int. max }}$ is found as: $\Delta y_{\text {int. max }}=\left(\left(\Delta_{i j \mathrm{ADC}}\right)_{\max }\right)_{\max }$. This parameter is the maximum difference between the curve 2 and the curve 1 in Fig. 3 . Actually, for calculating $n_{\text {int.LSB }}$ (17), the real ADC response is compared with the approached ADC response from curve 3 in Fig. 3. Therefore the ADC error systematic component (8) has to be corrected as

$$
\Delta_{\text {syst. } j \mathrm{ADC}}=m_{j \mathrm{ADC}}-f\left(m_{j V}\right)
$$

where $f\left(m_{j V}\right)=\left(m_{j V}-m_{q V}\right) \frac{m_{p \mathrm{ADC}}}{m_{p V}-m_{q V}} ; j=q \cdots p$, and the parameter used practically is

$\left(\Delta y_{\text {int. max }}\right)^{\prime}=\left(\left(m_{j \mathrm{ADC}}-f\left(m_{j V}\right)+\Delta_{\text {rand. } i j \mathrm{ADC}}\right)_{\max }\right)_{\max } \cdot$

The $\left|\Delta y_{\max }\right|-\left|\Delta y_{\min }\right|$ is found for $j=q \cdots p$ as

$$
\begin{aligned}
\left|\Delta y_{\max }\right|-\left|\Delta y_{\min }\right|= & \left|A_{j \mathrm{ADC}}-A_{(j-1) \mathrm{ADC}}\right|_{\max } \\
& -\left|A_{j \mathrm{ADC}}-A_{(j-1) \mathrm{ADC}}\right|_{\text {min }} .
\end{aligned}
$$

The values (17) written in the terms of the new approach are shown at the top of the page.

\section{REFERENCES}

[1] IEEE Standard for Performance Measurements of A/D and D/A Converters for PCM Television Video Circuits, no. 746, Oct. 15, 1984.

[2] E. G. Soenen, P. M. Vanpeteghem, H.-C. Lin, S. Narayan, and J. T. Cummings, "A framework for design and testing of analog integrated circuits," IEEE Trans. Instrum. Meas., vol. 39, pp. 890-893, Dec. 1990.

[3] T. M. Souders, R. Flach, and T. C. Wong, "An automatic test set for the dynamic characterization of A/D converters," IEEE Trans. Instrum. Meas., vol. IM-32, pp. 180-186, Mar. 1983.

[4] C. Clayton, J. A. Mcclean, and G. J. Mccarra, "FFT performance testing of data acquisition systems," IEEE Trans. Instrum. Meas., vol. IM-35, pp. 212-215, June 1986.

[5] C. A. Young, "An enhanced method for characterizing succesive approximation converters," IEEE Trans. Instrum. Meas., vol. 39, pp. 335-339, Apr. 1990.

[6] A. Brandolini and A. Gandelli, "Testing methodologies for analog-todigital converters," IEEE Trans. Instrum. Meas., vol. 41, pp. 595-603, Oct. 1992. 
[7] E. D. Koltik, G. D. Muginov, and G. P. Tsyvirko, "Calibration of measuring and computational systems for analysis of random signal parameters," in IMEKO-88, Applications 88-D53, 1988, pp. 573-580.

[8] M. F. Toner and G. W. Roberts, "A BIST for a SNR, gain tracking, and frequency response test of a sigma-delta ADC," IEEE Trans. Circuits Syst., vol. 42, pp. 1-15, Jan. 1995.

[9] "Integrated circuits, data book supplement." Tucson, AZ: Burr-Brown Corp., vol. 33b, sec. 9, 1990.

[10] "Data acquisition and linear devices databook." Santa-Clara, CA: National Semiconductor, 1989.

Galia D. Muginov received the M.Sc. degree from the Leningrad/St. Petersburg Institute of Electrical Engineering, Russia, in 1968, and the Ph.D. and D.Sc. degrees in electrical engineering from the D. I. Mendeleev Institute of Metrology, St. Petersburg, in 1974 and 1989, respectively.

She was a Junior and then a Senior Scientist at the D. I. Mendeleev Institute of Metrology from 1972 to 1989. In 1990 and 1991 she was a Professor of the Department of Metrology at the North-West Polytechnic Institute St.-Petersburg. From 1992 to 1995 she was a Scientific Researcher at the Center for Technological Education, Holon, Israel. She joined the University of Toronto, Toronto, Ont., Canada, in 1996, where she is now a Visiting Professor in the Department of Electrical and Computer Engineering. She is the author of 12 inventions, a State Standard, and a State Recommendation of Russia. She has published more than 30 papers. Her research interests are highspeed digital signal processing systems working in real-time (modeling, data acquisition, and data processing algorithms, test methods, and techniques); simulation of complex signals with controlled parameters; and up-to-date applied metrology of sophisticated electronic equipment for measuring, control, and testing.
Anastasios N. Venetsanopoulos (S'66-M'69-SM'79-F'88) received the B. Eng. degree from the National Technical University of Athens, Greece, in 1965, and the M.S., M.Phil., and Ph.D. degrees in electrical engineering from Yale University, New Haven, CT, in 1966, 1968, and 1969, respectively.

He joined the University of Toronto, Toronto, Ont., Canada, in September 1968; he has been a Professor there since 1981. He has served as Chairman of the Communications Group and as Associate Chairman of the Department of Electrical and Computer Engineering. He was on research leave at the Imperial College of Science and Technology, the National Technical University of Athens, the Swiss Federal Institute of Technology, the University of Florence and the Federal University of Rio de Janeiro, and was Adjunct Professor at Concordia University. He has served as a Lecturer in over 130 short courses to industry and continuing education programs and as Consultant to numerous organizations; he is a contributor to 24 books and has published over 550 papers on digital signal and image processing and digital communications.

He has served as Chairman on numerous boards, councils and technical conference committees including IEEE committees such as the Toronto Section (1977-1979) and the IEEE Central Canada Council (1980-1982). He was President of the Canadian Society for Electrical Engineering and Vice President of the Engineering Institute of Canada (1983-1986). He was a Guest Editor and Associate Editor for numerous IEEE journals and the Editor of the Canadian Electrical Engineering Journal (1981-1983). He is a member of the IEEE Communications, Circuits and Systems, Computer, and Signal Processing Societies, as well as a member of Sigma XI, the Technical Chamber of Greece, the European Association of Signal Processing, the Association of Professional Engineers of Ontario (APEO) and Greece. He was elected Fellow of the IEEE "for contributions to digital signal and image processing," and Fellow of EIC "for contributions to electrical engineering," and was awarded an Honorary Doctorate by the National Technical University of Athens, in October 1994. 